\title{
Effects of Interfacial Features on Yield Strength of Particle Reinforced Metal Matrix Composites
}

\author{
Jingjing Chen ${ }^{\mathrm{a}}$, Qun hui ${ }^{\mathrm{b}}$, Chunmei $\mathrm{LI}^{\mathrm{c}}$, Xiao Lid ${ }^{\mathrm{d}}$, Dongyuan Shao and Nanpu Cheng ${ }^{\mathrm{f}}$ \\ Faculty of Materials and Energy, Southwest University, Chongqing, China

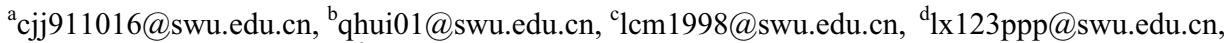 \\ esdy1988719@swu.edu.cn, ${ }^{\mathrm{f}}$ Corresponding author: cheng_np@swu.edu.cn
}

\begin{abstract}
The effective model assumption based on the average field theory has been modified and extended to investigate the effects of interfacial parameters including the bonding strength and interfacial thickness on the yield strength of particle reinforced composites. The formulation is applied to a model case of $\mathrm{SiC}$ particle reinforced $\mathrm{Al}$ matrix composite. The theoretical results agree well with the experimental ones of the $\mathrm{SiC} / \mathrm{Al}$ composite produced by a PM route. The modified theoretical model can effectively predict the interfacial behavior and provide the preparation of $\mathrm{SiC} / \mathrm{Al}$ composites with scientific foundation to control the interfaces.
\end{abstract}

Keywords: Interfacial feature; PRMCs; Interfacial bonding strength; Interfacial thickness

\section{Introduction}

Particle reinforced metal matrix composites have the advantages of high density-strength, density-modulus, thermal stability and internal friction over their counterparts, i.e. the metals or alloys, can be widely used as structure materials or functional materials and efforts have been done on preparing this sort of composites[1-3]. From these experiments one can find that the interface features will significantly affect the mechanical properties and failure mechanisms as well as the strength of the composites[4]. In order to improve the wettability or to restrain the undesirable interfacial reaction between $\mathrm{Al}$ matrix and $\mathrm{SiC}$ particle, the reinforcements $\mathrm{SiC}$ particles are often coated by $\mathrm{Cu}$ or $\mathrm{Ni}$, etc[5]. The surface oxidation of $\mathrm{SiC}$ particles is another simple way in surface treatment technique. In both of these surface treatments, there exists an interface layer in thickness of several tens of nanometers between the matrix and the reinforcements[6]. When the original $\mathrm{SiC}$ particles are used as reinforcements, the atomic diffraction of $\mathrm{Al}$ matrix into the $\mathrm{SiO}_{2}$ layer on the $\mathrm{SiC}$ particle surfaces also forms an amorphous layer[7].Such materials can be viewed as three phase composites, and the interphase will affect the properties of the composites. At the same time, theoretical studies on interfacial behavior based on the micromechanics in the particle reinforced composites are also fundamental and important questions, which can give us a deep investigation in the strengthening and failure mechanisms of the composites and 
provide scientific basis for practical preparation[8].

In this work, interfacial parameters including the bonding strength and thickness are introduced to modify the effective model assumption (EMA) model[8] (belong to the interphase model) and the effects on the properties of $\mathrm{SiCp} / \mathrm{Al}$ are studied. The theoretical results have been compared with our experimental results to examine the rationality of the current modified theoretical model.

\section{Theoretical Model of PRMCs}

\subsection{Preventative unit of PRMCs}

In the particulate reinforced composites, a thin interphase layer intervenes between a particle and the matrix, which has the same profile as the particle. For convenience in theoretical derivation and calculation, the particle is often considered as spherical with a diameter $r$, see Fig.1 (a), and the interface with thickness of $\delta$ together with the particle is thought of as an effective assemble/element (i.e. an effective reinforcement). Furthermore, the matrix with an embedding particle may be considered as a new effective element representing the particle composites, see Fig.1 (b). All of the particle, matrix and interphase are isotropic. For the perfectly bonded interface particulate composites, the most effective medium approach based on generalizations of the Eshelby method can be used. If the properties of the particle, interface and matrix are known, the properties of the effective reinforcement can be calculated through the Mori average field theory, and then the properties of the effective element of particle composites can be determined.
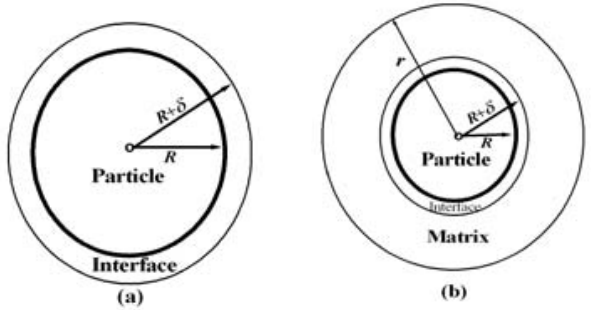

Fig. 1. EMA of PRMCs (a) Reinforcement and Interface and (b) Preventative Cell of PRMCs

To mainly discuss the effects of interface thickness and interface bonding strength on the effective reinforcement and to compare the theoretical results with our experiment, here $\mathrm{SiC}$ particles are taken as the reinforcement particle (surely, other reinforcement particles are also likely).

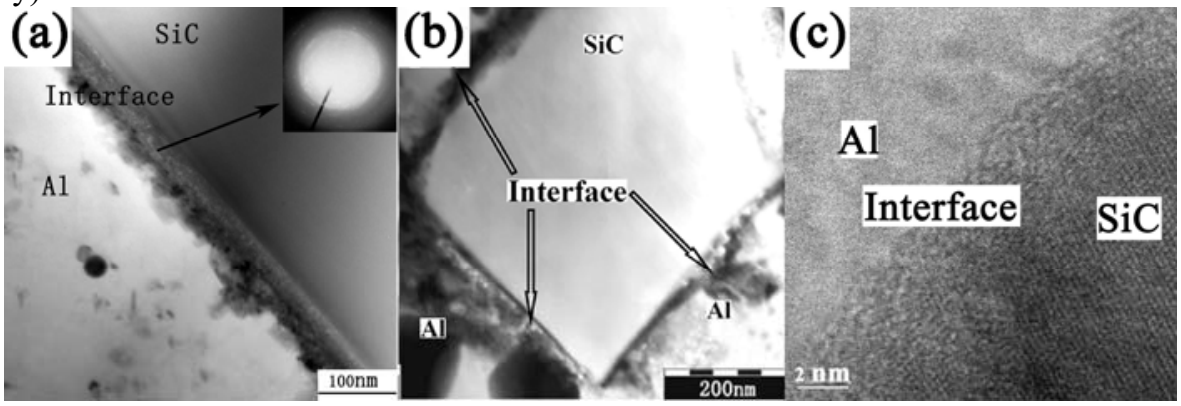

Fig. 2. Typical interfaces of $\mathrm{SiCp} / \mathrm{Al}$ composites reinforced by (a) original $\mathrm{SiC}$ particles; (b) surface-coated SiC particles; (c) oxidized, acid picked and alkaline cleaned SiC particles 
In Fig.2, Our experimental results indicate that the interface layer of the oxidized, acid picking and alkaline cleaned $\mathrm{SiC}$ particle reinforced $\mathrm{Al}$ composites are mainly $\mathrm{Al}$; the interface of the original $\mathrm{SiC}$ particle reinforced $\mathrm{Al}$ composites is mainly $\mathrm{Al}$ and $\mathrm{SiO}_{2}$; While the $\mathrm{SiC}$ particles surface were coated with $\mathrm{K}_{2} \mathrm{ZrF}_{4}$, the interface is mainly composed of $\mathrm{K}_{2} \mathrm{ZrF}_{4}$ [7]. When the over-oxidized $\mathrm{SiC}$ particles are used, the interface is a $\mathrm{SiO}_{2}$ layer in thickness of tens of nanometers[6].

TABLE.1 PROPERTIES OF SIC PARTICLES, INTERFACE AND METAL MATRIX

\begin{tabular}{cccc}
\hline & $\mathrm{SiC}$ & $\mathrm{Al}$ & $\mathrm{SiO} / \mathrm{K} 2 \mathrm{ZrF} 6$ \\
\hline Elastic modulus $(\mathrm{GPa})$ & 450 & 70 & \\
Poisson ratio & 0.17 & 0.33 & 0.25 \\
\hline
\end{tabular}

Then the physical properties of the interface, $\mathrm{Al}$ matrix and $\mathrm{SiC}$ particles are summarized in Table.1, which will be used in the theoretical calculation. The Poisson ratio of the interface maybe is not perfectly accurate, but it can help to analyze its effects. The relative bonding strength of interface here is expressed by $q=E_{\mathrm{i}} / E_{\mathrm{f}}$, a non-dimensional parameter. Then $q$ and $\gamma$ can predict the effects of interfacial features on the effective reinforcement. The case of $q=1$ implies that there is no interface displacement to jump and the interface is perfectly bonded with the reinforcement. Zero value of $q$ implies that the interface tractions do not exist and the reinforcement is fully debonded from the matrix. The values of $0<q<1$ define an imperfect interface cohesion, which lies between two extreme cases mentioned above.

\section{Effective Bulk Modulus}

For the perfectly bonded interface, the theoretical results of the effective reinforcement can be got from the work proposed by Hashin[9], as shown in Fig. 3. In the following formula, $k, \mu, E, v$ and $Q^{-1}$ represent the bulk modulus, shear modulus, elastic modulus, Poisson ration and internal friction, respectively. The subscripts $\mathrm{p}, \mathrm{i}$, and $\mathrm{m}$ refer to the particle, interphase, and matrix, respectively.

The interphase shell is thought as a part of the reinforcement. The volume ratio of the particle in the effective reinforcement is $V_{\mathrm{p}}=1 /(1+\gamma)^{3}$, where $\gamma$ is defined as a relative thickness of interface compared with the particle, $\gamma=\delta / R$. When the thickness of interface is much smaller than the radius of particle, namely, $\gamma \rightarrow 0$, then $V_{\mathrm{p}} \approx 1$. The effective bulk modulus $k^{*}$ for the effective reinforcement shown in Fig.1 (a) reads[9]

$$
k^{*}=k_{\mathrm{i}}+V_{\mathrm{p}}\left(\frac{1}{k_{\mathrm{f}}-k_{\mathrm{i}}}+\frac{3\left(1-V_{\mathrm{p}}\right)}{3 k_{\mathrm{i}}+4 \mu_{\mathrm{i}}}\right)
$$

\section{Effective Shear modulus}

Christensen and Lo[10] have obtained the condition for getting the shear modulus of the effective model with interphase by solving the equation:

$$
A\left(\frac{\mu^{*}}{\mu_{i}}\right)^{2}+B\left(\frac{\mu^{*}}{\mu_{i}}\right)+D=0
$$




\subsection{Effects of Interfacial Parameters on Yield Stress of PRMCs}

\subsubsection{Effective Medium Assumption Model for Yield Stress of PRMCs with Perfect interface}

In Ref.[11],the EMA model was used to simulate the uniaxial stress-strain curve of $\mathrm{SiCp} / \mathrm{Al}$ during the tensile test. The microstructural changes in the composites due to the presence of $\mathrm{SiC}$ particles were considered, whereas the effects of interfaces have been treated by averaging through the experimental results. Here, the effects of interfaces on microstructures of $\mathrm{SiCp} / \mathrm{Al}$ composites are no longer to be detailed, while only the effects of interface thickness and interface bonding strength on the yield stress are addressed. Based on the EMA model (see Fig.1, but the interfaces are not considered, namely, the reinforcements are perfectly boded with metal matrix without interphases),

$\left\{\begin{array}{l}\frac{k^{\mathrm{C}}-k^{\mathrm{m}}}{3 k^{\mathrm{C}}+4 \mu^{\mathrm{ms}}}=f \frac{k^{\mathrm{P}}-k^{\mathrm{m}}}{3 k^{\mathrm{p}}+4 \mu^{\mathrm{ms}}} \\ \frac{\mu^{\mathrm{C}}-\mu^{\mathrm{ms}}}{\mu^{\mathrm{C}}+y^{\mathrm{s}}}=f \frac{\mu^{\mathrm{P}}-\mu^{\mathrm{ms}}}{\mu^{\mathrm{P}}+y^{\mathrm{s}}}\end{array},\left\{\begin{array}{l}\bar{\varepsilon}_{k k}=\frac{3 k^{\mathrm{m}}+4 \mu^{\mathrm{ms}}}{3 k^{\mathrm{C}}+4 \mu^{m s}} \varepsilon_{k k}^{\mathrm{m}}, \quad \bar{\sigma}_{k k}=\frac{3 k^{\mathrm{m}}+4 \mu^{\mathrm{ms}}}{3 k^{C}+4 \mu^{m s}} \frac{k^{\mathrm{C}}}{k^{m}} \sigma_{k k}^{\mathrm{m}}, \\ \bar{\varepsilon}_{i j}^{\prime}=\frac{\mu^{\mathrm{ms}}+y^{\mathrm{s}}}{\mu^{\mathrm{C}}+y^{\mathrm{s}}} \varepsilon_{i j}^{\prime \mathrm{m}}, \quad \bar{\sigma}_{i j}^{\prime}=\frac{\mu^{\mathrm{ms}}+y^{\mathrm{s}}}{\mu^{\mathrm{C}}+y^{\mathrm{s}}} \frac{1}{\mu^{\mathrm{ms}} \mu^{\mathrm{C}}} \sigma_{i j}^{\prime \mathrm{m}},\end{array}\right.\right.$

with $y^{\mathrm{s}}=\frac{\mu^{\mathrm{ms}}\left(9 k^{\mathrm{m}}+8 \mu^{\mathrm{ms}}\right)}{6\left(k^{\mathrm{m}}+2 \mu^{\mathrm{ms}}\right)}$, where $k^{P}, \mu^{P}$ are of the bulk and shear moduli of the

reinforcements. $k_{\mathrm{c}}$ and $k_{\mathrm{m}}$ represent bulk modulus of the composites and the metal matrix; $V_{\mathrm{p}}$ is the volume fraction of reinforcements and $\mu_{\mathrm{ms}}$ is the secant modulus of matrix.

$\bar{\varepsilon}$ and $\bar{\sigma}$ are the average stress and strain in the represent element of composites, and $\varepsilon^{m}$ and $\sigma^{m}$ are the stress and strain in matrix, respectively.

\subsubsection{Effective Medium Assumption Model for Yield Stress of PRMCs with imperfect interface.}

$\mathrm{Ju}$ et al. have established the micromechanical model for particulate reinforced composites with imperfectly bonded interfaces[12]. The effective bulk and shear moduli, respectively, are given by

$$
\left\{\begin{array}{l}
k_{\mathrm{c}}=k_{\mathrm{m}}\left\{1+\frac{30\left(1-v_{\mathrm{m}}\right)\left(3 \lambda_{1}+2 \lambda_{2}\right) V_{\mathrm{P}}}{3 \xi_{1}+2 \xi_{2}-10\left(1+v_{\mathrm{m}}\right)\left(3 \lambda_{1}+2 \lambda_{2}\right) V_{\mathrm{P}}}\right\} \\
\mu_{\mathrm{c}}=\mu_{\mathrm{m}}\left\{1+\frac{30\left(1-v_{\mathrm{m}}\right) \lambda_{2} V_{\mathrm{P}}}{\xi_{2}-4\left(4-5 v_{\mathrm{m}}\right) \lambda_{2} V_{\mathrm{P}}}\right\}
\end{array}\right.
$$

Where $\xi_{1}, \xi_{2}, \lambda_{1}$ and $\lambda_{2}$ are functions of $k^{*}$ and $\mu^{*}$, the effective bulk modulus and effective shear modulus, respectively, and $V_{\mathrm{P}}$ represents the volume fraction of the reinforcement particles.

When the formed interfaces in the particle composites are very thin, namely $\gamma=\delta / R \rightarrow 0$, their volume is relatively small, which implies that the volume fraction of the effective reinforcement approximately equate to that of the particles in the composites. However, if the interphase is thick and can not be ignored, the volume fraction of the effective reinforcement in the composites should be represented by $V_{\mathrm{P}}^{\prime}=\frac{4 \pi(R+\delta)^{3} / 3}{4 \pi r^{3} / 3}=V_{\mathrm{P}} \times(1+\delta / R)^{3}$. Noting that the interface bonding strength 
together with the interface thickness affects properties of the particle composites, two cases, namely the volume of reinforcements containing and without containing the volume of interphase, should be respectively discussed. The materials parameters used in the theoretical simulation are shown in Table.1.

In order to consider the effects of interfacial features on the yield strength of composites, $k^{P}$ and $\mu^{P}$ in Eq.(3) should be replaced by the effective bulk modulus $k^{*}$ and shear modulus $\mu^{*}$ of the effective represent element with interfaces being considered. And $k^{\mathrm{C}}$ and $\mu^{\mathrm{C}}$ in Eq.(3) should be replaced by $k_{\mathrm{C}}$ and shear modulus $\mu_{\mathrm{C}}$ in Eq.(4). For convenient calculation, two assumptions have been addressed: (1) the reinforcements are perfectly elastic, and (2) when the materials justly become to yield, the secant modulus roughly equates to the shear modulus, namely, $\mu_{m s} \approx \mu_{m}$. Noting that the loading transfer between metal matrix and reinforcements carries out through the interfaces, the different interfacial features will affect the loading transfer. The effective Mises strain formula $\sigma_{\mathrm{e}}=\sqrt{\frac{3}{2} \sigma_{i j}^{\prime} \sigma_{i j}^{\prime}}$ is used in the calculation.

\subsubsection{Effects of Interface on Yield Stress of SiCp/Al composites}

Fig. 3 shows the effects of interfacial features and volume fraction of SiC particles on the yield strength of $\mathrm{SiCp} / \mathrm{Al}$. The case that the interfacial volume is contained in the reinforcements is plotted in the left column, while the the interfacial volume is not contained in the reinforcements is plotted in the right column. $\sigma_{\mathrm{Y}}^{\mathrm{c}}$ and $\sigma_{\mathrm{Y}}^{0}$ represent the yield strengths of composites and metal matrix, respectively. From the left column, it was found the yield strength increases from a same min to a same max value with the bonding strength increasing. However, it decreases with the interface thickness increasing. When $q=0$ (fully debonded interfaces), the relative yield strengths for the cases of different volume fraction of reinforcements are same to the corresponding volume fraction, which reveals the reinforcements together with the interfaces have no enhancing functions in the composites. When $q=1$ (perfectly bonded interfaces), the yield strengths of composites, compared with the metal alloy, have been enhanced about by $0.08,0.10,0.125$ and 0.15 for the different volume fractions $(10 \%, 12 \%, 15 \%$ and $18 \%)$ of reinforcements due to the load transferring mechanism. For the case that the interface volume is not contained in the reinforcements, the relative yield strength also increases with the bonding strength increasing and decreases with the interface thickness increasing, which is similar to the former case. But it decreases more at $q=0$ while increases higher at $q=1$. The theoretical results in the right column compared with that of the left column reveal that if the surface coated particles are used as reinforcements, only the well bonded interfaces can improve the mechanical properties. In fact, it is difficult to get the strongly bonded interfaces due to the thicker interface layers. If the particle surface coating is necessary to avoid the interfacial reaction between matrix and reinforcements in experiment, the rational way to control the interface is to get the thinner coating layer. 

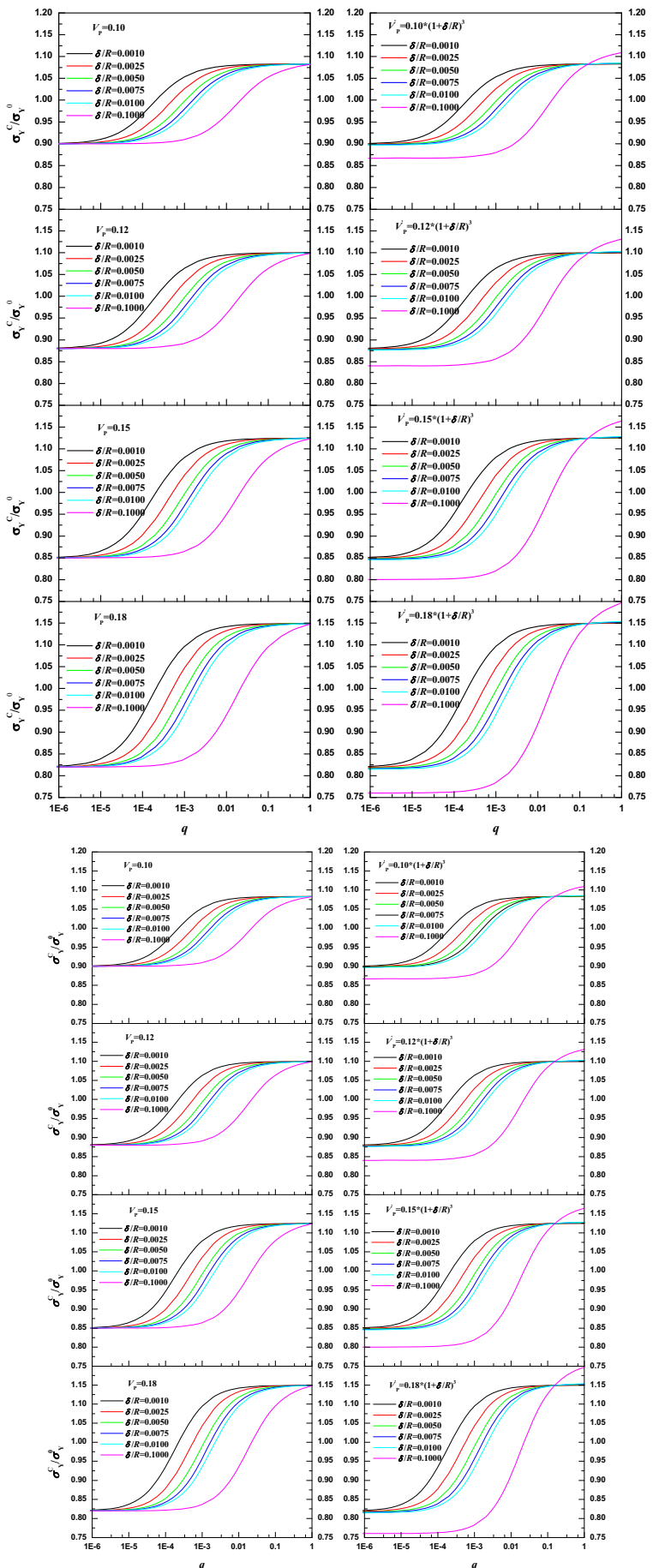

(a)

(b)

Fig. 3. Effects of interfacial features on yield strength of $\operatorname{SiCp} / \mathrm{Al}$ (a) $v_{i}=0.33$; (b) $v_{i}=0.25$ 


\section{Discussion}

The $\mathrm{SiC} / \mathrm{Al}$ interface is not only a critical technique in preparing $\mathrm{SiCp} / \mathrm{Al}$ composites but also an important and difficult question in theoretical studies. The interfacial features including interface thickness and interface bonding strength will strongly influence the properties of composites. In this paper, two parameters, e.i. the relative interface thickness and the relative bonding strength, have been introduced into the modified effective represent element of particle reinforced composites. These two parameters can describe the different interfaces in the as-received $\mathrm{SiCp} / \mathrm{Al}$ composites. Through the detailed calculation, the thicker or undesirable bonded interfaces will decrease the yield strength. The thicker or undesirable bonded interface is not beneficial to the loading transfer, which bonds to resulting in the decreased yield strength. When the oxidized, acid pickling and alkaline cleaned $\mathrm{SiC}$ particles were used as reinforcements, compared with the original $\mathrm{SiC}$ particles, the interfaces in $\mathrm{SiCp} / \mathrm{Al}$ composites produced by $\mathrm{PM}$ route was very thin and the reinforcement were well strongly bonded with matrix. Correspondingly, the composites have the higher strength. However, the interfaces in the surface-coated SiC particles reinforced $\mathrm{SiCp} / \mathrm{Al}$ composites were weakly bonded due to the defects such as cavities existing in the interfaces, which resulted in the sharply decreased strength. Based on experiments, when the reinforcements are surface coated, the thicker the coatings, the easier defects such as cavities are formed in interfaces during the drying process, which weakens the interface bond. In short, the thicker surface coatings often results in the weaker interface bonding strength and further deteriorate the mechanical properties of composites.

\section{Conclusions}

Based on the theoretical calculations through micromechanics, the effects of interfacial features on the yield strength of $\mathrm{SiCp} / \mathrm{Al}$ have been investigated, and some conclusions can be drawn as follows: (1) the parameterized micromechanics model can predict the properties of particle reinforced composites well, and the theoretical calculation can explain the current experimental results; (2) when the interface volume is contained in the reinforcements, the yield strength of particle reinforced composites increases with the interface bonding strength increasing, and decreases with the interfaces changing thicker; (3) when the interface volume is not considered in the reinforcements, the yield strength approximately increases with the interface bonding strength increasing. If the interface bonding strength is weak, the thinner interfaces will have the higher yield strength. For the strongly bonded interfaces, the thicker interfaces will result in the lower yield strength; (4) in experiment, consideration should be given to the higher yield strength. Hence, from the theoretical and experimental results, it is more reasonable to appropriately raise the volume fraction of reinforcements. If the surface coating is necessary to improve the wettability between matrix and reinforcements, the interfaces should be thin.

\section{Acknowledgements}

Subject supported by the National Natural Science Foundation of China (Grant No. 51171156) and the Fundamental Research Funds for the Central Universities of Ministry of Education of China (Grant No. XDJK2014C008). 


\section{References}

1. P. Divecha, S. G. Fishman and S. D. Karmarkar, Silicon carbide reinforced aluminum-a formable composite, J. Met.. 33 (1981) 12-17.

2. A. Ibrahim, F. A. Mohamed and E. J. Lavernia, Particulate reinforced metal matrix composites-a review, J. Mater. Sci.. 26 (1991) 1137-1156.

3. D. J. Lloyd, Particle reinforced aluminium and magnesium matrix composites, Int. Mater. Rev.. 39 (1994) 10-23.

4. M. Vedabu and E. Garibold, Influence of interface properties on mechnical behavior of particle reinforced metal matrix composite, Mater. Sci. Tech.. 10 (1994) 132-136.

5. T. P. D. Rajan, R. M. Pillai and B. C. Pai, Review Reinforcement coatings and interfaces in aluminium metal matrix composites, J. Mater. Sci.. 33 (1998) 3491-3503.

6. J. C. Lee, J. P. Ahn, J. H. Shim and Z. L. Shi, Control of the interface in SiC/Al composites, Scripta. Mater.. 41 (1999) 895-900.

7. N. P. Cheng, C. M. Li, Effect of particle surface treatment on the microstructure and property of SiCp/AA6066 composite produced by powder metallurgy, Mat. Sci. Eng. A. 517 (2009) 249-256.

8. S. H. Nie and C. Basaran, A micromechanical model for effective elastic properties of particulate composites with imperfect interfacial bonds, Int. J. Solids. Struct.. 42 (2005) 4179-4191.

9. Z. Hashin, The spherical inclusion with imperfect interfac, J. Appl. Mech-t. ASME.. 58 (1991) 444-449.

10. R. M. Christensen and K. H. Lo, Solutions for effective shear properties in three phase sphere and cylinder models, J. Mech. Phys. Solids.. 27 (1979) 315-330.

11. C. W. Nan and D. R. Clarke, The influence of particle size and particle fracture on the elastic/plastic deformation of metal matrix composites, Acta Mater. 44 (1996) 3801-3811.

12. J. W. Ju and T. M. Chen, Micromechanics and effective moduli of elastic composites containing randomly dispersed ellipsoidal inhomogeneities, Acta. Mechanica. 103 (1994) 103-121. 